\title{
Stratospheric variability and tropospheric annular-mode timescales
}

Article

Published Version

Simpson, I. R., Hitchcock, P., Shepherd, T. G. and Scinocca, J. F. (2011) Stratospheric variability and tropospheric annularmode timescales. Geophysical Research Letters, 38 (20). L20806. ISSN 0094-8276 doi: https://doi.org/10.1029/2011GL049304 Available at https://centaur.reading.ac.uk/31554/

It is advisable to refer to the publisher's version if you intend to cite from the work. See Guidance on citing.

To link to this article DOI: http://dx.doi.org/10.1029/2011GL049304

Publisher: American Geophysical Union

All outputs in CentAUR are protected by Intellectual Property Rights law, including copyright law. Copyright and IPR is retained by the creators or other copyright holders. Terms and conditions for use of this material are defined in the End User Agreement.

\section{www.reading.ac.uk/centaur}

\section{CentAUR}

Central Archive at the University of Reading 
Reading's research outputs online 


\title{
Stratospheric variability and tropospheric annular-mode timescales
}

\author{
I. R. Simpson, ${ }^{1}$ P. Hitchcock, ${ }^{1}$ T. G. Shepherd, ${ }^{1}$ and J. F. Scinocca ${ }^{2}$ \\ Received 15 August 2011; revised 28 September 2011; accepted 29 September 2011; published 29 October 2011.
}

[1] Climate models tend to exhibit much too persistent Southern Annular Mode (SAM) circulation anomalies in summer, compared to observations. Theoretical arguments suggest this bias may lead to an overly strong model response to anthropogenic forcing during this season, which is of interest since the largest observed changes in Southern Hemisphere high-latitude climate over the last few decades have occurred in summer, and are congruent with the SAM. The origin of this model bias is examined here in the case of the Canadian Middle Atmosphere Model, using a novel technique to quantify the influence of stratospheric variability on tropospheric annular-mode timescales. Part of the model bias is shown to be attributable to the too-late breakdown of the stratospheric polar vortex, which allows the tropospheric influence of stratospheric variability to extend into early summer. However, the analysis also reveals an enhanced summertime persistence of the model's SAM that is unrelated to either stratospheric variability or the bias in model stratospheric climatology, and is thus of tropospheric origin. No such feature is evident in the Northern Hemisphere. The effect of stratospheric variability in lengthening tropospheric annular-mode timescales is evident in both hemispheres. While in the Southern Hemisphere the effect is restricted to late-spring/early summer, in the Northern Hemisphere it can occur throughout the winter-spring season, with the seasonality of peak timescales exhibiting considerable variability between different 50 year sections of the same simulation. Citation: Simpson, I. R., P. Hitchcock, T. G. Shepherd, and J. F. Scinocca (2011), Stratospheric variability and tropospheric annularmode timescales, Geophys. Res. Lett., 38, L20806, doi:10.1029/ 2011 GL049304.

\section{Introduction}

[2] The annular modes (AM's) are the dominant modes of variability in the extra-tropical circulation in both the troposphere and stratosphere [e.g., Thompson and Wallace, 2000; Kushner, 2010]. They are also the preferred response of the extra-tropical circulation to many different climate forcings. Indeed, the predicted circulation response to climate change projects strongly onto the tropospheric AM's in each hemisphere [e.g., Miller et al., 2006].

[3] The fluctuation-dissipation (F-D) theorem relates the magnitude of a forced AM-like response to the timescale of natural AM variability [Ring and Plumb, 2008]. Longer

\footnotetext{
${ }^{1}$ Department of Physics, University of Toronto, Toronto, Ontario, Canada.

${ }^{2}$ Canadian Centre for Climate Modelling and Analysis, Environment Canada, Victoria, British Columbia, Canada.

Copyright 2011 by the American Geophysical Union. 0094-8276/11/2011GL049304
}

timescales imply a larger magnitude response to an external forcing. The explanation of this result lies in the fact that both the persistence of the AM and the magnitude of the response to a forcing are determined by the strength of the same internal positive feedbacks which, in the case of tropospheric AM's, are predominantly due to interactions between synoptic scale eddies and the mean flow [Lorenz and Hartmann, 2001]. Therefore, in order to accurately predict future climate change, a General Circulation Model (GCM) must correctly simulate the internal tropospheric feedbacks and exhibit the correct timescale of AM variability associated with such feedbacks.

[4] While GCM's generally capture AM timescales and their seasonal evolution, a consistent bias is that tropospheric timescales are too long in the SH summer [Gerber et al., 2008, 2010]. If this is related to biases in tropospheric feedbacks, then by the F-D theorem this implies that the models may be inaccurately predicting the magnitude of future extra-tropical circulation changes in that season. This is important because the largest observed changes in SH highlatitude climate over the last few decades have occurred in summer, and are congruent with the SAM [Fogt et al., 2009].

[5] Aside from internal tropospheric feedbacks, tropospheric AM persistence may be influenced by external forcing on intraseasonal timescales [Keeley et al., 2009]. One important intraseasonal forcing on tropospheric AM's comes from stratosphere-troposphere coupling. Baldwin et al. [2003] first demonstrated that in seasons when large amplitude, long timescale AM variability occurs in the stratosphere, the tropospheric AM timescales are correspondingly longer. Because stratosphere-resolving GCM's tend to exhibit a too-late breakdown of the SH stratospheric vortex [Butchart et al., 2011], their stratospheric AM variability continues too late into the summer, which could account for the overly strong tropospheric AM persistence during this season. However, Gerber et al. [2008] noted that some of the models that exhibit this latter bias do not have a fully resolved stratosphere and so this is unlikely to be the complete story.

[6] It is therefore important to quantify the impact of stratospheric variability on tropospheric AM timescales in GCM's and isolate the component of the AM timescale that is associated with internal tropospheric feedbacks. Purely statistical diagnostics are ambiguous because tropospheric AM timescales may be affected by other factors that vary seasonally alongside stratospheric variability, such as tropospheric jet structure. In this study, we address this issue by suppressing the zonal-mean stratospheric variability in a GCM in a way that leaves the zonal-mean climatology unchanged. This provides an unambiguous quantification of the role of stratospheric variability in the seasonal evolution of tropospheric AM timescales, including the relative importance 


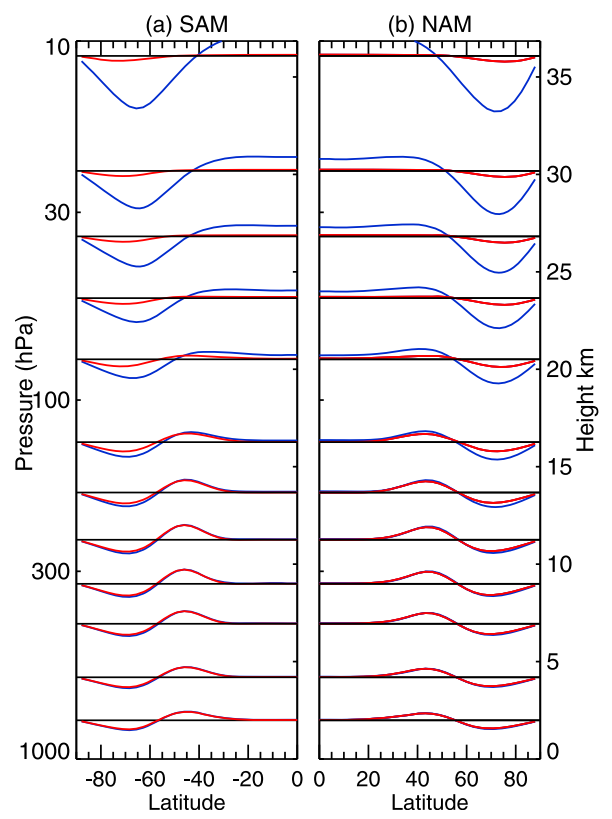

Figure 1. Latitudinal structure of the (a) SAM and (b) NAM. Calculated by projecting $\Phi^{\prime}$ (in $\mathrm{km}$ ), scaled by cos(latitude), onto the NAM/SAM index, displacing by the height of the relevant pressure level after multiplying by 3 to increase visibility. Blue $=$ FREE, red $=$ NUDG.

of stratospheric model biases on the SH summer tropospheric AM biases.

\section{Model Experiments, Data and Timescale Diagnostic}

[7] The model used is the dynamical version of the Canadian Middle Atmosphere Model (CMAM) [Scinocca et al., 2008]. This is a comprehensive, high top GCM with T63 horizontal resolution and 71 levels in the vertical stretching from the surface to $0.0006 \mathrm{hPa}(\sim 100 \mathrm{~km})$. This version of the model does not include interactive chemistry or have a quasi-biennial oscillation. Time slice repeated annual cycle simulations are run with seasonally varying climatological SSTs and sea-ice and greenhouse gas concentrations held fixed at 1990's values. A 100 year free running control simulation, denoted "FREE" and a 100 year nudged simulation, denoted "NUDG" have been performed. In NUDG the zonal mean vorticity, divergence and temperature are nudged toward the seasonally varying climatology of FREE in the stratosphere and above. Because the model is nudged to its natural climatology, the nudging exerts no net torque in the time average which would otherwise affect the tropospheric climatology. This nudging is performed as a simple relaxation, $-K(p)\left(X-X_{\text {clim }}\right) / \tau_{N}$, on the zonal mean, where $X$ is the instantaneous value of the zonal mean and $X_{\text {clim }}$ is the seasonally varying zonal-mean climatology. The timescale, $\tau_{N}$, is 6 hours and the factor $K$ allows for a vertical variation in the nudging strength which is increased linearly from 0 below $64 \mathrm{hPa}$ to 1 above $28 \mathrm{hPa}$. Another experiment in which the nudging was switched on abruptly at $100 \mathrm{hPa}$ produced very similar results to the higher altitude, linear nudging profile presented here. The climatology was generated from the 6 hourly spectral output from FREE with smoothing applied by retaining only up to the fourth harmonic of the annual cycle. FREE and NUDG, therefore, have very similar zonal mean seasonally varying climatologies but in NUDG the zonal-mean variability in the stratosphere is damped out by the nudging process. Planetary waves can still freely propagate in the stratosphere but their influence on the zonal mean is suppressed. Note that histograms of the daily vertical Eliassen-Palm flux at levels throughout the troposphere and up to the midstratosphere are very similar in FREE and NUDG, and therefore the nudging does not appear to have a significant impact on planetary wave reflection. An additional 100 year simulation, denoted "NUDG_ERA", in which the stratosphere is nudged in a similar manner but to the 1989-2008 ERA-interim climatology [Dee et al., 2011], is used to determine the effect of the climatological timing of the $\mathrm{SH}$ vortex breakdown on tropospheric timescales. ERA-interim only extends to $1 \mathrm{hPa}$ so nudging is not applied above that level. The model results are compared with daily, zonal mean geopotential from ERA-interim reanalysis from 19792010 at 18 vertical levels from the surface to $10 \mathrm{hPa}$.

[8] The primary diagnostic used is the decorrelation timescale $(\tau)$ of the first empirical orthogonal function (EOF) of daily zonal mean geopotential $(\Phi)[$ Baldwin and Thompson, 2009], in each hemisphere at each vertical level. The EOF was calculated following the procedure outlined by Gerber et al. [2010]. For each day and each pressure level, the global mean $\Phi$ is subtracted from $\Phi$ at each latitude and the resulting geopotential anomaly is de-seasonalised giving the input, $\Phi^{\prime}$, for the EOF calculation. The reanalysis data is also detrended using a linear trend. The first EOF of $\Phi^{\prime}$ and the corresponding Northern or Southern Annular Mode index, $I$, which has unit variance, is calculated on each level, using data from 0 to $90 \mathrm{~N}$ or 0 to $90 \mathrm{~S}$, applying a $\sqrt{\cos (\text { latitude) }}$ weighting to account for the decrease in area toward the pole.

[9] The autocorrelation function (ACF) of $I$ is calculated as a function of day of the year $d$ and lag $l$ by:

$$
A C F(d, l)=\frac{\sum_{y=1}^{N_{y}-1} I(d, y) I(d+l, y)}{\sqrt{\sum_{y=1}^{N_{y}-1} I(d, y)^{2} \sum_{y=1}^{N_{y}-1} I(d+l, y)^{2}}},
$$

where $y$ is the year and $N_{y}$ is the number of years of data. This is smoothed over a 181 day window using a Gaussian filter with a full width at half maximum of around 42 days (standard deviation $\sigma=18$ ). The e-folding timescale $(\tau)$ of this smoothed ACF is then calculated using a least squares fit to an exponential up to a lag of 50 days. This method differs from that outlined by Mudryk and Kushner [2011] in that the Gaussian smoothing is done after the calculation of the ACF. However, the results are robust to whichever method is used. Two different measures of statistical significance are used and these are outlined in Appendix A.

\section{Results}

\subsection{The Southern Annular Mode (SAM)}

[10] The SAM structure of FREE, shown in Figure 1a (blue curves), closely resembles the observed SAM [cf. Gerber et al., 2010, Figure 4]. The nudging (red curves) considerably reduces the SAM variability in the stratosphere, as expected, whereas in the troposphere, the structures have been largely unaffected. The timescales of the 
(a) ERA, 1979-2010

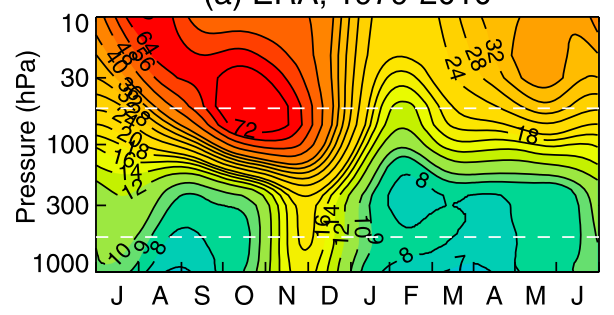

(b) FREE, 100y

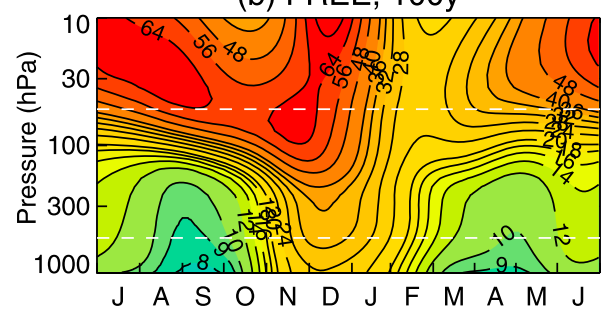

(c) Red $=52 \mathrm{hPa}$, Blue $=531 \mathrm{hPa}$

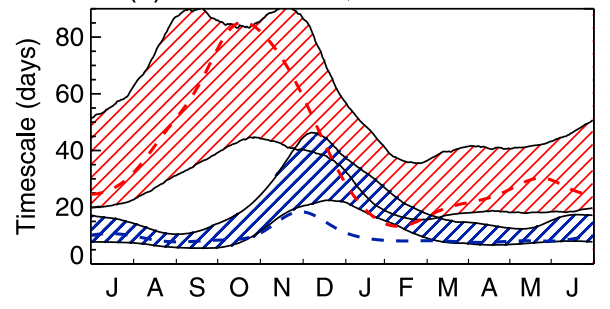

Figure 2. SAM timescales (in days) for (a) ERA-interim reanalysis and (b) FREE (note the non-linear contour interval in this and subsequent plots). (c) SAM timescales on the $52 \mathrm{hPa}$ (red) and $531 \mathrm{hPa}$ (blue) levels. These two levels are indicated by the white dashed lines in Figures $2 \mathrm{a}$ and $2 \mathrm{~b}$. The dashed lines show the reanalysis timescales and these can be compared with the shaded region which represents the $95 \%$ confidence interval obtained from 1000, 32 year bootstrapping samples from FREE. When the dashed lines occur outside of the shaded region, there is less than a $2.5 \%$ chance that the reanalysis timescales could be obtained with a 32 year model sample.

SAM in FREE are first compared with those of the reanalysis in Figure 2. The reanalysis plot (Figure 2a) reproduces the results of previous studies [e.g., Baldwin et al., 2003] and demonstrates enhanced timescales in the stratosphere during SH spring associated with the large amplitude variability that can occur due to anomalously early or late breakdowns of the SH polar vortex. During November and December, when the long timescale variability reaches down to around $100 \mathrm{hPa}$, the tropospheric timescales increase substantially from about 8 days to 16 days. The seasonal evolution of timescales in FREE (Figure 2b) shows qualitatively similar features although some differences do exist. The significance of these differences can be assessed in Figure $2 \mathrm{c}$ which shows the observed timescales and the bootstrapping confidence interval on a 32 year FREE sample. Two levels are shown: $52 \mathrm{hPa}$, to represent the stratosphere and $531 \mathrm{hPa}$, to represent the troposphere. In the stratosphere, long timescale variability continues too late in the model and the model timescales are significantly longer than the reanalysis in January and February. In the troposphere a large bias exists in DJF. This is the common model bias discussed in section 1. Timescales are much too long in this season and even the lowest limit of the bootstrapping estimate is around twice the observed value.

[11] The contribution of stratospheric zonal mean variability to the seasonal evolution of tropospheric SAM timescales can now be assessed by comparing FREE with NUDG (Figure 3). The FREE-NUDG difference (Figure 3a) confirms that stratospheric variability does indeed enhance the tropospheric SAM timescales in the SH spring/early summer. The tropospheric timescales are examined in more detail in Figure 3c. From November to January, stratospheric variability acts to roughly double tropospheric timescales from around 14 days in NUDG to 30 days in FREE. However, NUDG has a rather dramatic seasonal variation of its own. Figure $3 \mathrm{~b}$ shows that timescales are enhanced in November to February, even in the absence of stratospheric variability. Comparison of the green curve in Figure $3 \mathrm{c}$ with the reanalysis (dashed blue line) demonstrates that even when the contribution to SAM persistence from the stratosphere is absent, the tropospheric SAM timescales in summer are biased long compared to the observations. This implies that a significant proportion (approximately half) of the bias in $\mathrm{SH}$ tropospheric timescales during DJF is not associated with the biases in stratospheric zonal mean variability.

[12] One possibility is that the residual tropospheric bias is related to stratospheric climatology, i.e., to the too late breakdown of the SH vortex. To examine this, the NUDG_ERA SAM timescales are shown in Figure 4. This demonstrates

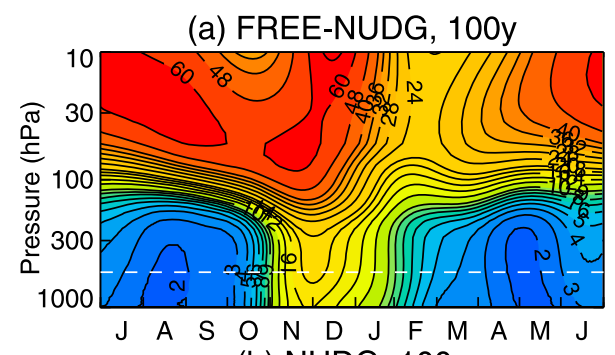

(b) NUDG, 100y

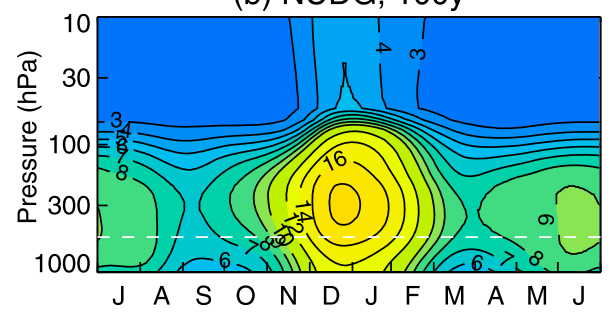

(c) Blue=FREE, Green=NUDG

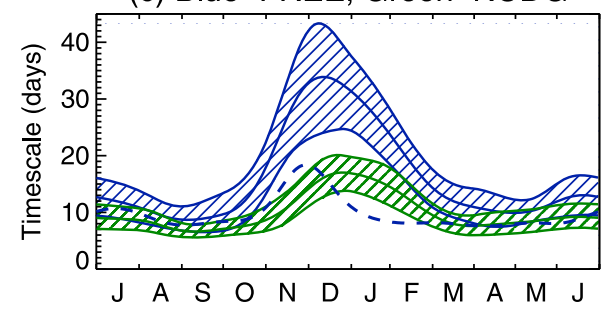

Figure 3. (a) Difference in SAM timescales (in days) between FREE and NUDG, (b) SAM timescales for NUDG and (c) SAM timescales on the $531 \mathrm{hPa}$ level. The $531 \mathrm{hPa}$ level is indicated by the white dashed line in Figures 3a and $3 \mathrm{~b}$. In Figure $3 \mathrm{c}$ shading $=95 \%$ confidence interval on the timescales for FREE (blue) and NUDG (green). Blue dashed line $=$ reanalysis timescales. 


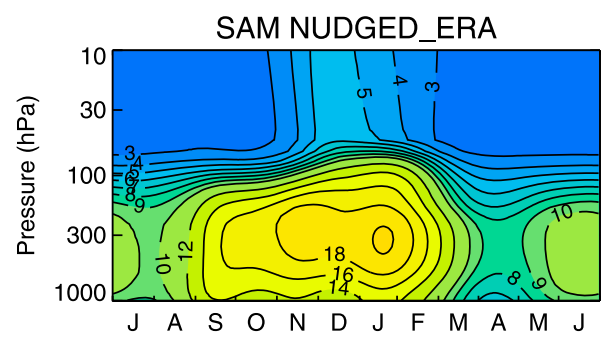

Figure 4. SAM timescales (in days) for the NUDG_ERA simulation.

that having the correct timing of the breakdown of the $\mathrm{SH}$ vortex does not alleviate the bias. We therefore conclude that the residual bias in tropospheric AM timescale seen in NUDG during summer is of tropospheric origin.

\subsection{The Northern Annular Mode (NAM)}

[13] We now repeat the analysis for the NAM timescales. The NAM structures of FREE and NUDG are shown in Figure $1 \mathrm{~b}$ and show similar features to their SH counterparts. The FREE, NUDG and FREE-NUDG timescales are shown in Figures 5a-5c. FREE has maximum timescales in the stratosphere in the winter season, associated with variability in the strength of the stratospheric polar vortex. The tropospheric timescales also show a maximum in the winter coincident with the maximum timescale in the lowermost stratosphere (as first noted by Baldwin et al. [2003]). However, in contrast to the $\mathrm{SH}$, there is no strong seasonality in the NUDG timescale in the NH implying that the seasonality in FREE arises from the downward influence of stratospheric zonal mean variability. Indeed, Figure 5c demonstrates that stratospheric variability acts to approximately double the tropospheric NAM timescales in winter.

(a) FREE, 100y

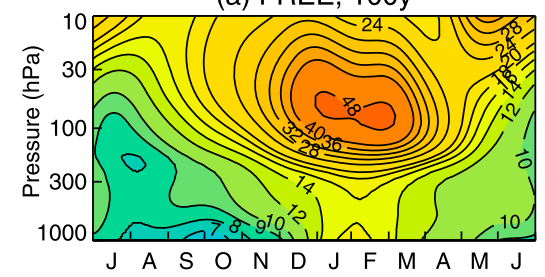

(b) NUDG, 100y
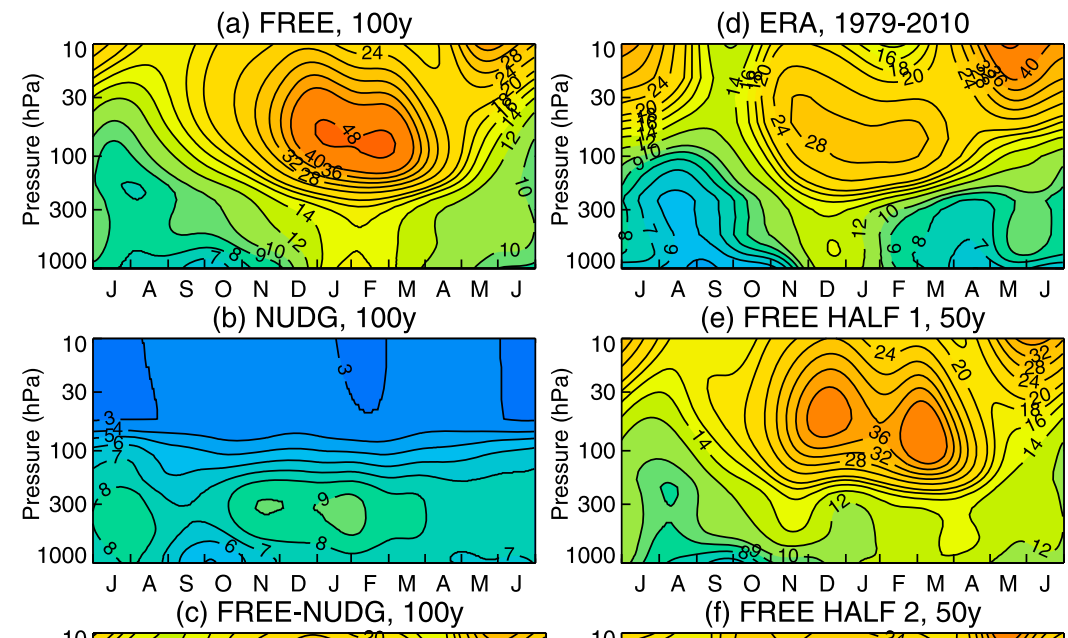

(e) FREE HALF 1, 50y

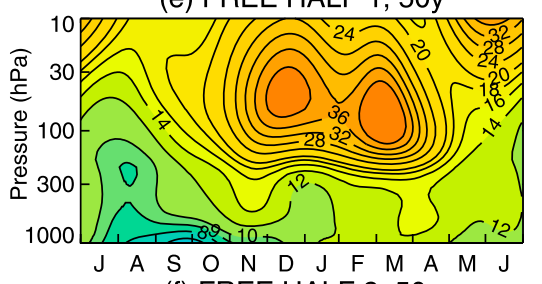

(f) FREE HALF 2, 50y

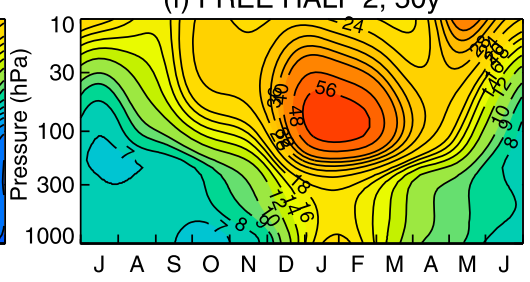

Figure 5. NAM timescales (in days) for (a) FREE, (b) NUDG, (c) FREE-NUDG, (d) ERA-interim, (e) the first 50 year half of FREE and (f) the second 50 year half of FREE. 
virtually all of the seasonality in AM timescales arises from the downward influence of stratospheric AM variability. Earlier numerical studies such as Norton [2003] and Gerber and Polvani [2009] demonstrated a potential role for stratospheric variability in lengthening tropospheric timescales by adding a perturbation to either reduce or enhance stratospheric variability. However, the perturbations applied in each of those studies also had a significant impact on both the tropospheric and stratospheric climatology and so the attribution to changes in stratospheric variability was not clear-cut. By using a novel approach to keep the climatology the same while damping the stratospheric variability, it is demonstrated that in CMAM the presence of stratospheric variability approximately doubles the tropospheric timescales in the active seasons.

[16] Furthermore, it has been possible to isolate the contributions to the bias in SH summer AM timescales that is common among GCM's. While there is a role for the too late breakdown of the SH vortex, a considerable proportion of the bias, in particular the extension of the enhanced timescales into the summer months, appears to be tropospheric in origin. A possible candidate for giving rise to this bias is the tropospheric jet latitude. The CMAM jet is located too far equatorward in DJF (a bias that is common among GCM's [Fyfe and Saenko, 2006]) and several authors have shown that when jets are located at lower latitudes, their timescale of variability increases [e.g., Kidston and Gerber, 2010]. In fact, nudging to ERA-interim in the stratosphere acts to shift the jet further equatorward, particularly in SON, through changes in lower stratospheric temperature. The fact that NUDG_ERA shows enhanced timescales in SON compared to $\bar{N} U D G$ provides further evidence that tropospheric jet structure likely plays a role in this bias.

[17] Whether or not jet structure is the most important factor, it can be concluded that the SH summer AM timescale bias is associated with some form of bias in the strength of the internal tropospheric feedbacks. Via the F-D theorem this implies that, all else being equal, models are likely to overestimate the SAM response to forcings in this season and thus overestimate the magnitude of the predicted poleward shift of the jet in future climate change scenarios. It is also possible that removing the bias in tropospheric feedbacks would also act to reduce the influence of the stratospheric biases. A greater understanding of the cause of this bias is required and alleviating it is likely to be important in the correct prediction of future $\mathrm{SH}$ summertime climate change.

\section{Appendix A: Estimation of Statistical Significance}

[18] The reanalysis data is 32 years in length. To assess the significance of differences between model and reanalysis, bootstrapping with replacement is used to generate 1000, 32-year samples from FREE and provide a 95\% confidence interval on the $\tau$ calculated from FREE with a 32 year sample. Where the reanalysis lies outside of this interval there is less than a $2.5 \%$ chance that the model and reanalysis are representing the same $\tau$.

[19] To provide a confidence interval on the modeled timescales from a simulation of length $N_{y}$, the ACF of each year up to $N_{y}-1$ was calculated. Gaussian smoothing of each ACF was then performed resulting in a smoothed estimate of the ACF of each day of each year individually. The standard deviation, $\sigma$, of these ACF's for each day of the year was then used to provide a $95 \%$ confidence interval on the ACF at each lag $\left(C_{95}(d, l)=\left(\sigma(d, l) / \sqrt{N_{y}-1}\right) t_{N y-2}\right)$, where $t_{N y-2}$ is the t value corresponding to $95 \%$ significance with $N_{y}-2$ degrees of freedom. A normal distribution of the ACF's has therefore been assumed. The upper and lower bounds on the ACF are then given by $A C F(d, l) \pm C_{95}(d, l)$ and the upper and lower bounds on $\tau$ calculated from them. This is found to provide a good estimate when using a sample AR1 process of known timescale and comparing with the 95\% confidence limit calculated using a large number of such samples.

[20] Acknowledgments. This work was funded by the Natural Sciences and Engineering Research Council of Canada. We are grateful to Mike Neish for technical assistance, Michael Sigmond for providing some of the data used and two anonymous reviewers.

[21] The Editor thanks the two anonymous reviewers for their assistance in evaluating this paper.

\section{References}

Baldwin, M. P., and D. W. J. Thompson (2009), A critical comparison of stratosphere-troposphere coupling indices, Q. J. R. Meteorol. Soc., 135, 1661-1672.

Baldwin, M. P., D. B. Stephenson, D. W. J. Thompson, T. J. Dunkerton, A. J. Charlton, and A. O'Neill (2003), Stratospheric memory and skill of extended-range weather forecasts, Science, 301, 636-640, doi:10.1126/ science. 1087143

Butchart, N., et al. (2011), Multimodel climate and variability of the stratosphere, J. Geophys. Res., 116, D05102, doi:10.1029/2010JD014995.

Dee, D. P., et al. (2011), The ERA-interim reanalysis: Configuration and performance of the data assimilation system, Q. J. R. Meteorol. Soc., 137, 553-597, doi:10.1002/qj.828.

Fogt, R. L., J. Perlwitz, A. J. Monaghan, D. H. Bromwich, J. M. Jones, and G. J. Marshall (2009), Historical SAM variability. Part II: Twentiethcentury variability and trends from reconstructions, observations, and the IPCC AR4 models, J.Clim., 22, 5346-5365, doi:10.1175/ 2009JCLI2786.1.

Fyfe, J. C., and O. A. Saenko (2006), Simulated changes in the extratropical Southern Hemisphere winds and currents, Geophys. Res. Lett., 33, L06701, doi:10.1029/2005GL025332.

Gerber, E. P., and L. M. Polvani (2009), Stratosphere-troposphere coupling in a relatively simple AGCM: The importance of stratospheric variability, J. Clim., 22, 1920-1933.

Gerber, E. P., L. M. Polvani, and D. Ancukiewicz (2008), Annular mode time scales in the Intergovernmental Panel on Climate Change Fourth Assessment Report models, Geophys. Res. Lett., 35, L22707, doi:10.1029/2008GL035712.

Gerber, E. P., et al. (2010), Stratosphere-troposphere coupling and annular mode variability in chemistry-climate models, J. Geophys. Res., 115, D00M06, doi:10.1029/2009JD013770.

Keeley, S. P. E., R. T. Sutton, and L. C. Shaffrey (2009), Does the North Atlantic Oscillation show unusual persistence on intraseasonal timescales?, Geophys. Res. Lett., 36, L22706, doi:10.1029/2009GL040367.

Kidston, J., and E. P. Gerber (2010), Intermodel variability of the poleward shift of the austral jet stream in the CMIP3 integrations linked to biases in 20th century climatology, Geophys. Res. Lett., 37, L09708, doi:10.1029/2010GL042873.

Kushner, P. J. (2010), Annular modes of the troposphere and stratosphere, in The Stratosphere: Dynamics, Transport, and Chemistry, Geophys. Monogr. Ser., vol. 190, edited by L. M. Polvani, A. H. Sobel, and D. W. Waugh, pp. 59-91, AGU, Washington, D. C., doi:10.1029/ 2009GM000924.

Lorenz, D. J., and D. L. Hartmann (2001), Eddy-zonal flow feedback in the Southern Hemisphere, J. Atmos. Sci., 58, 3312-3327.

Miller, R. L., G. A. Schmidt, and D. T. Shindell (2006), Forced annular variations in the 20th century Intergovernmental Panel on Climate Change Fourth Assessment Report models, J. Geophys. Res., 111, D18101, doi:10.1029/2005JD006323.

Mudryk, L. R., and P. J. Kushner (2011), A method to diagnose sources of annular mode time scales, J. Geophys. Res., 116, D14114, doi:10.1029/ 2010JD015291 
Norton, W. A. (2003), Sensitivity of Northern Hemisphere surface climate to simulation of the stratospheric polar vortex, Geophys. Res. Lett., 30(12), 1627, doi:10.1029/2003GL016958.

Ring, M. J., and R. A. Plumb (2008), The response of a simplified GCM to axisymmetric forcings: Applicability of the fluctuation-dissipation theorem, J. Atmos. Sci., 65, 3880-3898.

Scinocca, J. F., N. A. McFarlane, M. Lazare, J. Li, and D. Plummer (2008), Technical note: The CCCma third generation AGCM and its extension into the middle atmosphere, Atmos. Chem. Phys., 8, 7055-7074.
Thompson, D. W. J., and J. M. Wallace (2000), Annular modes in the extratropical circulation. Part I: Month-to-month variability, J. Clim., 13, 1000-1016.

P. Hitchcock, T. G. Shepherd, and I. R. Simpson, Department of Physics, University of Toronto, 60 St. George St., Toronto, ON M5S 1A7, Canada. (isla@atmosp.physics.utoronto.ca)

J. F. Scinocca, Canadian Centre for Climate Modelling and Analysis, Environment Canada, PO Box 3065 STN CSC,Victoria, BC V8W 3V6, Canada. 